\title{
SIMPLICIAL STRUCTURE OF THE REAL ANALYTIC CUT LOCUS
}

\author{
MICHAEL A. BUCHNER
}

\begin{abstract}
ABSTRACr. This note shows how to generalize to arbitrary dimensions the result of S.B. Myers that the cut locus in a real analytic Riemannian surface is triangulable. The basic tool is Hironaka's theory of subanalytic sets.
\end{abstract}

In 1935 and 1936 there appeared in the Duke Mathematical Journal two interesting papers by Sumner B. Myers [3], [4] on the cut locus for a Riemannian manifold. He determined completely the local structure of the cut locus on a two-dimensional real analytic manifold. It turned out that for compact real analytic surfaces the cut locus is a one-dimensional finite simplicial complex. On the other hand in [4] he remarks that "it seems difficult to prove that the locus in the analytic $n$-dimensional case is homeomorphic to a finite $(n-1)$-dimensional complex". The argument that follows will establish this conjecture.

On the one hand we have Hironaka's theory [1], [2] of subanalytic sets which he proved to be triangulable. On the other hand Morse theory gives us a characterization of the cut locus in terms of the behavior of an analytic function on an analytic manifold fibered over the given manifold (the "energy function"). It seemed reasonable then to try to prove the cut locus is subanalytic. This is what the following argument establishes by rather simple reasoning.

Let $M$ be a compact analytic Riemannian manifold. Let $p \in M$. If $\gamma$ is a geodesic parametrized by arclength such that $\gamma(0)=p$ then the cut point of $(M, p)$ along $\gamma$ is defined to be the first point $\gamma\left(t_{0}\right)\left(t_{0}>0\right)$ such that for $t>t_{0}, \gamma$ no longer minimizes arclength from $p$ to $\gamma(t)$. The set of all such cut points as $\gamma$ varies over all possible geodesics from $p$ is denoted $C(p)$.

It is standard that $C(p)$ is characterized by $C(p)=\{x \in M \mid$ either $x$ is the first conjugate point on a length minimizing geodesic starting at $p$ and going through $x$ or there are at least two length minimizing geodesics from $p$ to $x$ \} [6].

Let $\Omega(M)$ be the space of piecewise analytic paths starting at $p$ and let $E: \Omega(M) \rightarrow \mathbf{R}$ (the energy function) be defined by $E(\gamma)=\int_{0}^{1}\|d \gamma(t) / d t\|^{2} d t$ (where we assume now that all paths are parametrized by the unit interval). Choose $\varepsilon>0$ so that whenever $d(x, y)<\varepsilon$ there is a unique geodesic from $x$ to $y$ of length $<\varepsilon$ and so that the geodesic depends analytically on the

Received by the editors May 12, 1976.

AMS (MOS) subject classifications (1970). Primary 53C20; Secondary 32B20, 32C05. 
endpoints $x$ and $y .(d(x, y)$ denotes the length from $x$ to $y$ induced by the Riemannian metric.)

Suppose the diameter of $M$ is $<\sqrt{s}$. Let $t_{0}, t_{1}, \ldots, t_{k}$, be a subdivision of $[0,1]$ so that each $t_{i+1}-t_{i}<\varepsilon^{2} / s$. Let $\Omega\left(t_{0}, t_{1}, \ldots, t_{k}\right)$ be the space of broken geodesics starting at $p$ (i.e. such that $\omega \in \Omega\left(t_{0}, t_{1}, \ldots, t_{k}\right)$ if and only if each $\omega \mid\left[t_{i}, t_{i+1}\right]$ is a geodesic). Let $\Omega\left(t_{0}, t_{1}, \ldots, t_{k}\right)^{s}$ be defined by $\Omega\left(t_{0}, t_{1}, \ldots, t_{k}\right)$ $\cap E^{-1}([0, s))$. It is easy to verify that if $\omega \in \Omega\left(t_{0}, t_{1}, \ldots, t_{k}\right)^{s}$ then

$$
d\left(\omega\left(t_{i}\right), \omega\left(t_{i+1}\right)\right)<\varepsilon
$$

so that we have a bijection

$$
\begin{aligned}
\Omega\left(t_{0}, t_{1}, \ldots, t_{k}\right)^{s} \rightarrow\left\{\left(x_{1}, \ldots, x_{k}\right) \in\right. & M \times M \times \cdots \times M \mid \\
& \left.\sum_{i=1}^{k} \frac{\left[d\left(x_{i-1}, x_{i}\right)\right]^{2}}{t_{i}-t_{i-1}}<s\right\} \text { where } x_{0}=p .
\end{aligned}
$$

REMARK. Many of the assertions concerning these path spaces can be found, with details, in Milnor [5].

The space $\Omega\left(t_{0}, \ldots, t_{k}\right)^{s}$ thus acquires an analytic structure and

$$
E \mid \Omega\left(t_{0}, \ldots, t_{k}\right)^{s}
$$

is an analytic map to $\mathbf{R}$. Let $\pi: \Omega\left(t_{0}, \ldots, t_{k}\right)^{s} \rightarrow M$ be the endpoint projection, i.e. $\pi(\omega)=\omega(1)$. Then $\pi$ is an analytic submersion.

In view of the characterization of the cut locus given earlier we have $C(p)=\left\{x \in M|E| \pi^{-1}(x)\right.$ has a degenerate minimum or at least two minima\}. (Here degenerate means the Hessian of $E \mid \pi^{-1}(x)$ at the minimum is not of full rank.)

Let us denote $\Omega\left(t_{0}, \ldots, t_{k}\right)^{s}$ by $B_{s}$. By $B_{s} \times_{M} B_{s}$ we mean the fibered product of $B_{s}$ with itself over $M$, i.e. $B_{s} \times_{M} B_{s}$ is the disjoint union $\cup_{x \in M^{2}} \pi^{-1}(x)$ $\times \pi^{-1}(x)$ with an obvious analytic manifold structure. The map $F: B_{s} \times_{M} B_{s}$ $\rightarrow \mathbf{R}$ given by $\left(b_{1}, b_{2}\right) \mapsto E\left(b_{1}\right)-E\left(b_{2}\right)$ is an analytic map and so the set $V \subset B_{s} \times_{M} B_{s}$ defined by $V=\left\{\left(b_{1}, b_{2}\right) \mid F\left(b_{1}, b_{2}\right)>0\right\}$ is semianalytic in $B_{s} \times_{M} B_{s}$.

Let $t$ be a number satisfying (diameter of $M)^{2}<t<s$. Then $B_{t} \times_{M} B_{t}$ is an open relatively compact subset of $B_{s} \times_{M} B_{s}$ and is also semianalytic. It follows that $V \cap\left(B_{t} \times_{M} B_{t}\right)$ is an open relatively compact semianalytic subset of $B_{s} \times_{M} B_{s}$.

At this point we need some facts about subanalytic sets (the basic reference being Hironaka [1]). Recall that if $\left(X, \theta_{X}\right)$ is a real analytic space $\left(\theta_{X}\right.$ is the structure sheaf) then $A \subset X$ is subanalytic if we can find real analytic spaces $V_{i j}(i=1, \ldots, k, j=1,2)$ and proper real analytic maps $g_{i j}: V_{i j} \rightarrow X$ such that $A=\cup_{i=1}^{k}\left\{g_{i 1}\left(V_{i 1}\right)-g_{i 2}\left(V_{i 2}\right)\right\}$. We wish to make use of the basic fact that the proper real analytic image of a subanalytic set is subanalytic. Although we will not necessarily be dealing with proper real analytic maps, we have the following substitute: Let $f:\left(X, \theta_{X}\right) \rightarrow\left(Y, \theta_{Y}\right)$ be a real analytic map between real analytic spaces where $X$ is paracompact and suppose 
$A \subset X$ is subanalytic and relatively compact; then $f(A)$ is subanalytic. To see this note that Hironaka proves that if $A \subset X$ is subanalytic and $X$ is paracompact there is a single real analytic space $\left(V, \theta_{V}\right)$ and a proper real analytic map $g$ such that $g(V)=\bar{A}$ (the closure of $A$ ). By compactness of $\bar{A}$ and properness of $g$ it follows that $f \circ g$ is proper. Moreover $g^{-1}(A)$ is subanalytic so $f \circ g\left(g^{-1}(A)\right)=f(A)$ is subanalytic.

Let $p_{1}$ denote projection of $B_{s} \times_{M} B_{s}$ on the first factor. We then obtain, in view of the above remarks, that $p_{1}\left(V \cap\left(B_{t} \times_{M} B_{t}\right)\right)$ is subanalytic in $B_{s}$. (Here we are using the fact that semianalytic sets are subanalytic.) The set $p_{1}\left(V \cap\left(B_{t} \times_{M} B_{t}\right)\right)$ consists of broken geodesic paths starting at $p$, having energy less than $t$, and for which there is another such path with the same endpoints and yet smaller energy. If $b_{1} \in p_{1}\left(V \cap\left(B_{t} \times_{M} B_{t}\right)\right)$ then there is some point $b_{2} \in B_{t}$ in $\pi^{-1}\left(\pi\left(b_{1}\right)\right) \cap B_{t}$ with $E\left(b_{2}\right)<E\left(b_{1}\right)$. Hence the complement of $p_{1}\left(V \cap\left(B_{t} \times_{M} B_{t}\right)\right)$ in $B_{t}$ consists of those points $b \in B_{t}$ such that $E \mid \pi^{-1}(\pi(b))$ is minimum at $b$ relative to $\pi^{-1}(\pi(b)) \cap B_{t}$. But since (diameter $M)^{2}<t$ we have that this complement consists exactly of $\mu=\left\{b \in B_{s}|E| \pi^{-1}(\pi(b))\right.$ is minimum at $\left.b\right\}$. This set $\mu$ is thus a subanalytic subset of $B_{s}$.

The subset $\mu \times_{M} \mu \subset B_{s} \times_{M} B_{s}$ is easily seen to be subanalytic. The diagonal of $B_{s} \times_{M} B_{s}$, which we denote by $\Delta$, is subanalytic. It follows that $\mu \times_{M} \mu-\Delta$ is subanalytic. As it is relatively compact (the energy of any minimum must be $\leqslant(\text { diameter } M)^{2}<s$ ) we observe that

$$
\left(\pi \times_{M} \pi\right)\left(\mu \times_{M} \mu-\Delta\right)
$$

is subanalytic in $M$, where $\pi \times_{M} \pi$ is the map from $B_{s} \times_{M} B_{s} \rightarrow M$ determined by $\pi$. This set is precisely the set of $x \in M$ such that $E \mid \pi^{-1}(x)$ has at least two minima.

Since $\pi$ is a submersion and $\mu$ is relatively compact it follows that $\mu$ is covered by a finite number of local products $U_{1}, U_{2}, \ldots, U_{k}$, i.e. each $U_{i}$ is diffeomorphic to $V_{i} \times D_{i}$ where $V_{i}$ is open in $M$ and $D_{i}$ is open in $\mathbf{R}^{q}$ (where $q$ is the dimension of the fiber of the map $\pi: B_{s} \rightarrow M$ ) and we have the commutative diagram

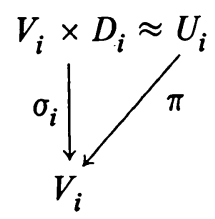

where $\sigma_{i}$ is projection on the first factor. The above isomorphism and $E$ define a function on $V_{i} \times D_{i}$ which we denote by $E_{i}=E_{i}(x, d)$. Let $H\left(E_{i}, x, d\right)$ denote the Hessian matrix of $E_{i, x}$ at $d$ where $E_{i, x}(d)=E_{i}(x, d)$. Then the set $\zeta_{i}=\left\{(x, d) \mid H\left(E_{i}, x, d\right)\right.$ has rank $\left.<q\right\}$ is semianalytic. We view $\zeta_{i}$ as a subset of $U_{i}$ and obtain a semianalytic subset $\zeta_{i} \cap \mu$ in $U_{i}$. These $\left\{\zeta_{i} \cap \mu\right\}$ piece together to give a semianalytic set in $B_{s}$ (observe that the rank of the Hessian is invariantly defined at a critical point). Finally because the $\cup_{i}\left(\zeta_{i} \cap \mu\right)$ is 
relatively compact in $B_{s}$ we obtain $\pi\left(\cup_{i}\left(\zeta_{i} \cap \mu\right)\right)$ is subanalytic in $M$. But this set is precisely the set $\left\{x \in M|E| \pi^{-1}(x)\right.$ has a degenerate minimum $\}$. We may now conclude that the cut locus itself is subanalytic.

\section{REFERENCES}

1. H. Hironaka, Subanalytic sets in number theory, algebraic geometry and commutative algebra, Papers in honor of Y. Akizuki, Kinokuniya, Tokyo, 1973, pp. 453-493.

2. - Triangulations of algebraic sets, Proc. Sympos. Pure Math., vol. 29, Amer. Math. Soc., Providence, R.I., 1975, pp. 165-185. MR 51 \# 10331.

3. Sumner B. Myers, Connections between differential geometry and topology. I: Simply connected surfaces, Duke Math. J. 1 (1935), 376-391.

4. - Connections between differential geometry and topology. II: Closed surfaces, Duke Math. J. 2 (1936), 95-102.

5. J. Milnor, Morse theory, Ann. of Math. Studies, no. 51, Princeton Univ. Press, Princeton, N.J., 1963. MR 29 \#634.

6. M. Buchner, Stability of the cut locus in dimensions $\leqslant 5$ (to appear).

Sonderforschungsbereich, Theoretische Mathematik, Beringstrasse 1, 5300 Bonn, FedERAL Republic of Germany 